\title{
Can Vertical Mixing from Turbulent Kinetic Energy Mitigate Coral Bleaching? An Application of High Frequency Ocean Radar
}

\author{
Diane D. DiMassa ${ }^{1}$, Malcolm L. Heron ${ }^{2}$, Alessandra Mantovanelli ${ }^{2}$, Scott F. Heron ${ }^{3}$, Craig Steinberg ${ }^{4}$ \\ 1. Massachusetts Maritime Academy, 101 Academy Drive, Buzzards Bay, MA 02532 USA ddimassa@maritime.edu \\ 2. Australian Coastal Ocean Radar Network (ACORN), Marine Geophysical Laboratory, James Cook University, Townsville, \\ Australia; 3. NOAA Coral Reef Watch (CRW) and Physics Dept, James Cook University (JCU), Townsville, Australia; \\ 4. Australian Institute of Marine Science (AIMS) and JCU, Townsville, Australia.
}

\begin{abstract}
Coral bleaching is an ecological response to stressful physical conditions observed to occur when strong insolation coupled with stratification of the water column leads to anomalous warming of the surface water. Stratfication requires calm winds, the absence of waves, and an absence of currents: conditions which result in limited mixing of the water column and thus confine heat due to insolation at the ocean surface. There is a strong need to identify which of the physical parameters are more significant at any given time and, more importantly, to monitor the physical parameters in near realtime to serve as a tool for long-term planning and management for marine parks and coastal waters. This paper reviews the contribution that currents make to mixing in the water column through the dissipation of turbulent kinetic energy and takes a further step to evaluate the use of surface current data to provide an index of vertical mixing. In this work, when the surface current speed is greater than a critical value, the water column is found to be vertically mixed even in the absence of wind or waves. A phased array HF Ocean Radar deployed in the southern part of the Great Barrier Reef provides a map of surface currents with high spatial resolution $(4 \mathrm{~km})$ every 10 minutes over the grid. These surface currents are used to predict vertical stratification and mixing which can then be used as an indication for conditions under which bleaching might occur.
\end{abstract}

\section{INTRODUCTION}

Tropical corals live in a symbiotic relationship with zooxanthellae that live within their tissues. These protozoa are photosynthetic and provide the coral with oxygen and necessary nutrients. The zooxanthellae also give the coral its color. Under stressful conditions, corals can undergo the natural ecological response of expelling their symbionts. This often results in a completely white appearance, hence the term 'bleaching.' However, prolonged periods without the protozoa lead to coral mortality. Temperature change, particularly an increase in temperature, is the most common index of coral bleaching in reef ecosystems [1]. When strong insolation is coupled with stratification in the water column, the surface waters can become anomalously warm, and this can contribute to significant coral stress. Water temperature stratification occurs when there are calm winds, the absence of waves, and low currents [2]. If, however, there is adequate vertical mixing due to wind-driven waves or currents, the heat due to insolation is distributed throughout the water column and, thus, any surface temperature increase is minimized. This paper considers only vertical mixing that results from tides and does not consider mixing due to waves or wind.

Tidal mixing results from boundary layer shear due to friction at the bottom of the water column. The horizontal velocity profile induces shear, and mixing is achieved through eddies in the vertical plane. This effect is strongest near the bottom where shears are greatest. However, in shallow water, where most tropical coral species are found, if strong currents exist, the boundary layer can reach the surface and mixing due to vertical eddies can be present throughout the entire water column. Vertical mixing is also impeded by stratification, and the turbulent kinetic energy (TKE) created by the eddies must be sufficient to overcome the potential energy of stratification. When the TKE dominates, the water is mixed and the risk of bleaching can be reduced. When the potential energy dominates, heat from solar insolation is trapped in the surface layer and the risk of bleaching is generally increased $[2,3]$.

A first step in determining conditions for coral bleaching is to evaluate the magnitude of the current needed to produce tidal mixing. Measured currents can then be compared to this required value, and the likelihood of mixing can then be determined. A data-based approach is to measure both speed and temperature throughout the water column and observe speeds when thermal stratification is, and is not, present. The required speed at the given location is then determined directly from these data. This information is then used to extrapolate required current speeds throughout the surrounding area as a function of bathymetry. In this work, when the surface current speed is greater than the critical value, the water column is assumed to be vertically mixed even in the absence of wind or waves. This critical value is established using data from two ADCP moorings which have collocated thermistors.

\section{OBSERVATIONS}

The area of study for this work is the Capricorn/ Bunker region of the southern Great Barrier Reef, and particularly the area surrounding Heron Island, (see Fig. 1). Two moorings, maintained by the Australia National Moorings Network (ANMN) as part of the Integrated Marine Observing System (IMOS), were deployed on the northern 

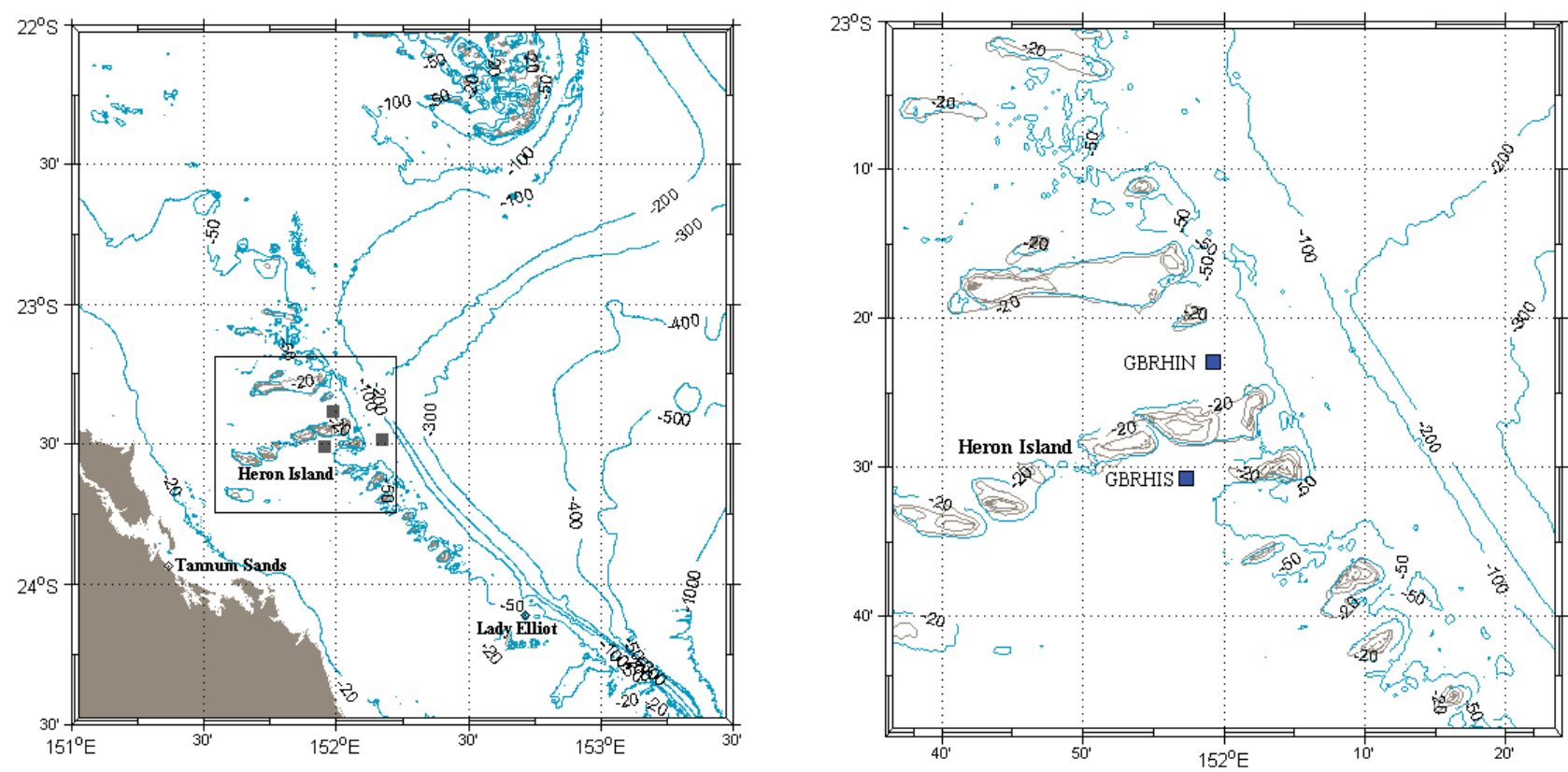

Figure 1. a). Capricorn/Bunker Region of Southern Great Barrier Reef. b). Enlargement of Heron Island Region as indicated in (a). Heavier lines indicate coastline or reef outline, lighter lines indicate bathymetry contours. Moorings GBRHIN and GBRHIS, North and South of Heron Island, respectively, are marked by squares. Twin WERA phased array HF radar stations at Tannum Sands and Lady Elliot Island are also shown. The radar station at Tannum Sands looks northeast $\pm 45^{\circ}$, whereas the station at Lady Elliot Island looks northwest $\pm 45^{\circ}$, and Heron Island lies approximately in the center of the coverage area.

and southern side of the island. Each mooring contained multiple SeaBird SBE39 Temperature Recorders to obtain temperature data at various depths throughout the water column and also contained an upward-looking RDI Workhorse ADCP to obtain current profiles. Temperature data taken by the ADCPs are also included in this analysis. Data from these instruments are freely available through IMOS. The mooring depths during the period of interest were $46 \mathrm{~m}$ and $48 \mathrm{~m}$ at the north (GBRHIN) and south (GBRHIS) sides of the island, respectively. As shown, GBRHIS is located in a more sheltered area, whereas GBRHIN is located in a channel between reefs.

Fig. 2 shows temperature data (a) North and (b) South of Heron Island for a 3-day period in August 2008. The raw temperature data from both ADCPs showed an offset and has been corrected. The correction is based on data measurements during a period of mixing when the temperature should be uniform throughout the water column. Offsets of $0.9^{\circ} \mathrm{C}$ and $-0.15^{\circ} \mathrm{C}$ were used for North and South, respectively. At GBRHIS, the overall temperature variability appears to follow the tidal cycle. However, it is not the value of the temperature that is important to this work; it is the degree of stratification as an indication of mixing. The data show that there are periods where the water column is completely thermally mixed, (for example 6AM and 6PM on 02 August 2008) and periods where it begins to stratify (for example 12AM and 12PM on 02 August 2008). At GBRHIN, the water column appears to be well-mixed throughout the entire time period, and the temperature variations only loosely follow the tidal cycle.
The temperature variability and stratification were then compared to current speeds, obtained from the ADCPs, at the corresponding depths. Surface currents were determined by the twin phased array WERA HF Ocean Radar Systems at Tannum Sands and Lady Elliot Island, which are managed by the Australian Coastal Ocean Radar Network (ACORN) of IMOS. The radars operate at a frequency of $8.340 \mathrm{MHz}$ and provide a map of surface currents with $4 \mathrm{~km}$ spatial resolution every 10 minutes over the coverage area of approximately 150 $\mathrm{km} \times 150 \mathrm{~km}$. Data are again freely available through IMOS. Fig. 3 shows a time series of surface current magnitude that corresponds to both the location of the GBRHIN and GBRHIS moorings and the time period shown in Fig. 2. Radar data, filtered only by eliminating noise spikes in currents above 1.8 $\mathrm{m} / \mathrm{s}$, are plotted as well as an hourly average. Hourly averages are not displayed if there are less than two data points in the hour of interest. The tidal signature is clearly seen in these data, and the hourly averaging provides sufficient smoothness to the time series. During mid-day on 03 August 2008, there was a short period where much of the radar data were not reliable. This is true throughout the coverage area, and the cause of this data loss has not been determined.

Fig. 4 shows the hourly averaged surface current speed from the HF radar superimposed on the current speeds determined by the ADCP for a) North (GBRHIN) and b) South (GBRHIS), respectively. Again, the tidal cycle is clearly visible at all depths. However, at certain periods, the surface current speed is greater than the speeds at depth and at other times, the surface current speed is less than the speeds at 


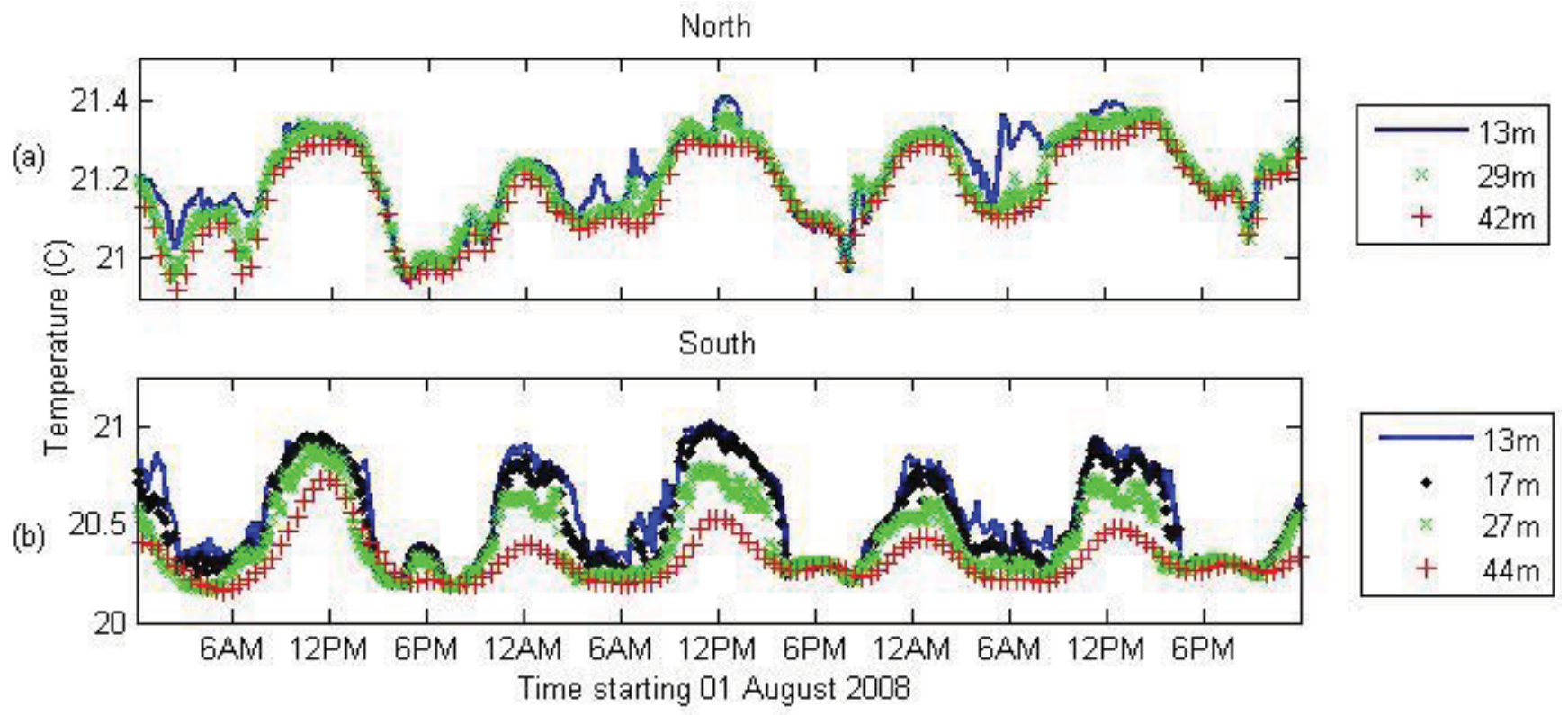

Figure 2. SeaBird and ADCP temperature data for 01-03 August 2008 for moorings a) North and b) South of Heron Island. Note different scales for temperature and that time is given in UTC.

(a)

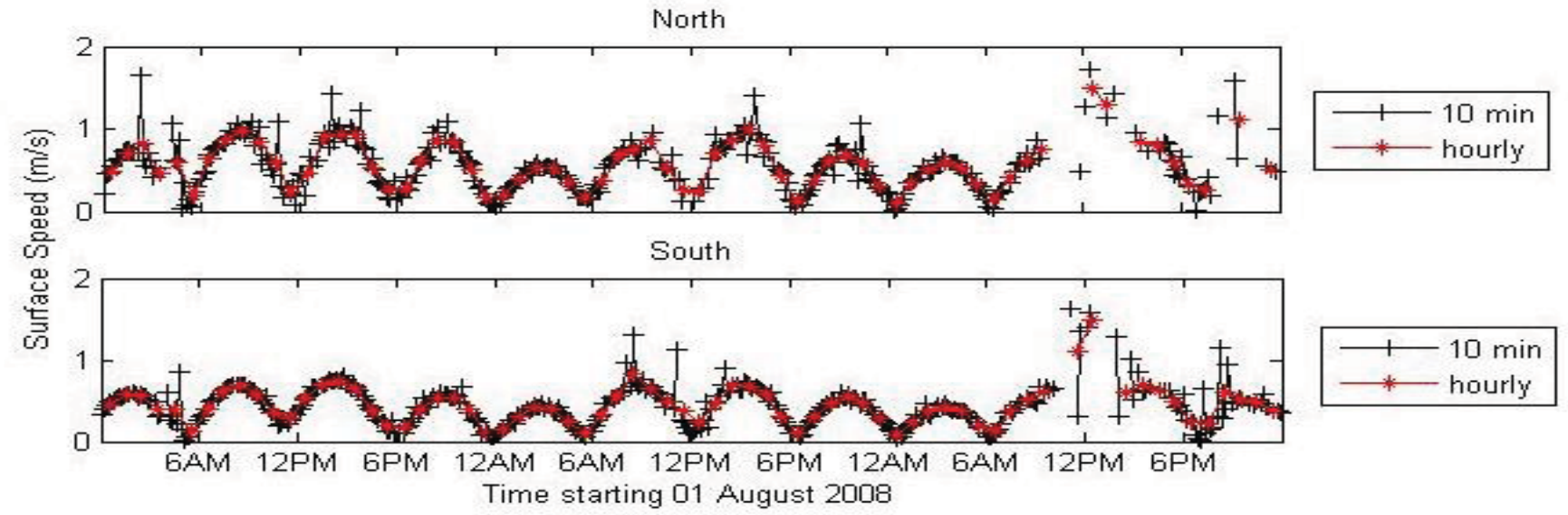

Figure 3. Surface current speed as determined by HF Radar for 01-03 August 2008 at mooring locations a) North and b) South of Heron Island. Note that time is given in UTC.

(a)

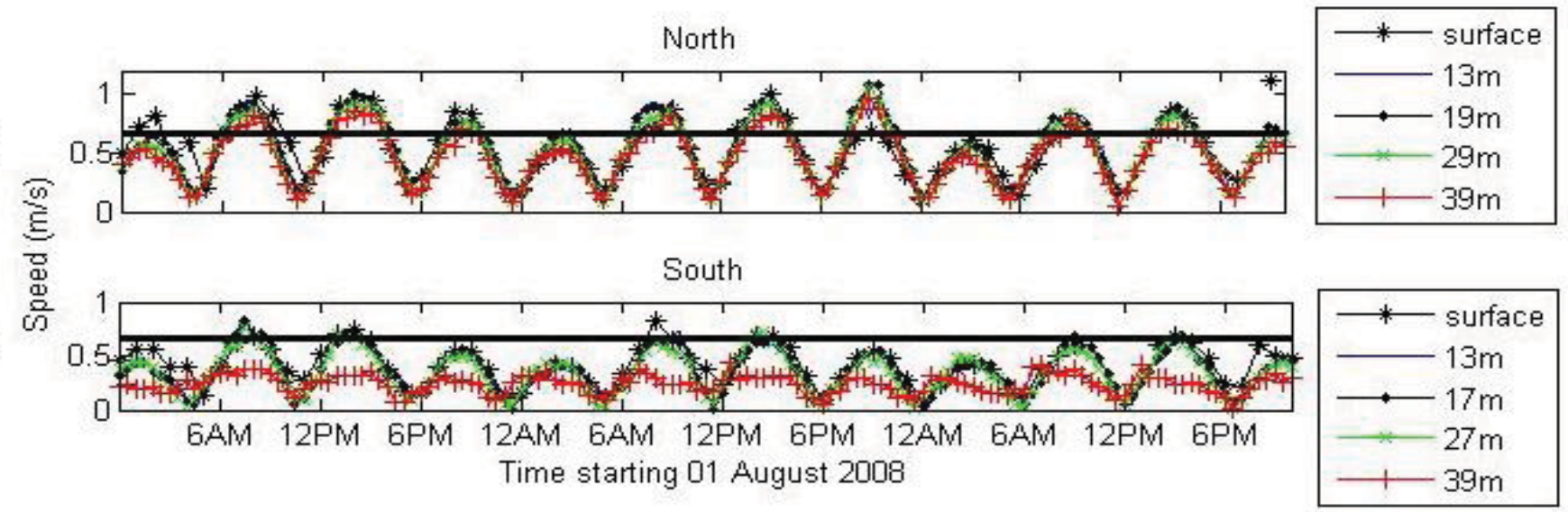

Figure 4. Current Speeds at surface and temperature logger depths for 01-03 August 2008 corresponding to moorings a) North and b) South of Heron Island. The solid black lines are the critical speeds above which turbulent kinetic energy dominates. Note that time is given in UTC. 
depth. Regardless, it is shown that the surface currents are a strong indicator of the currents throughout the water column.

\section{RESULTS}

A convenient index to evaluate the degrees of mixing and stratification involves a balance between the potential energy of stratification and the turbulent kinetic energy [4] The Simpson-Hunter parameter (SHP), defined as

$$
S H P=\log _{10} \frac{h}{U^{3}}
$$

where $\mathrm{h}$ is the water depth $(\mathrm{m})$ and $\mathrm{U}$ is the surface velocity $(\mathrm{m} / \mathrm{s})$, is determined by equating the turbulent kinetic energy dissipation with the energy required to destroy stratification through vertical mixing $[4,5]$. The literature shows that the Simpson-Hunter threshold for tidal mixing varies most often between 1.8 and 2.7 [6], depending highly on bathymetry, bottom topography, and seasonality. For this work, the temperature data of Fig. 2 were correlated with the current speed data of Fig. 4 to determine the speed required to induce mixing and destratify the water column. This speed and the mooring depth were then used to determine an appropriate value for the SHP threshold. For example, at GBRHIS the reduced speeds at 00:00 on 02 August 2008 correspond to the development of stratification, whereas in the six preceding hours, the higher speeds correlate with a well-mixed and nearly uniform temperature water column. These data were then used to determine that an appropriate Simpson-Hunter value for this area would be 2.2, which is within the range of values often cited in the literature. Further data analysis shows that the Simpson-Hunter value of 2.2 may actually be too low and thus the required velocity may represent a conservative value. However, for the spatial resolution available, it was determined that the approximation that resulted from the Simpson-Hunter parameter of 2.2 would be a sufficient starting value for this research.

Using this value, the critical velocity required to create a tidal mixing front throughout the region was determined from equation 1 and the depth. The bold horizontal lines in Fig. 4 indicate the critical velocities at the mooring locations. For GBRHIN, the value is $0.66 \mathrm{~m} / \mathrm{s}$ and for GBRHIS, the value is $0.67 \mathrm{~m} / \mathrm{s}$. Velocities above this value are assumed to produce vertical mixing, to distribute the heat due to insolation, and thus to minimize any temperature increase that might lead to bleaching. The data show that during this period of time, the region North of Heron Island experienced currents large enough to produce the onset of turbulent mixing due to a tidal front. These favorable conditions happen frequently over the 3-day period of study, and the temperature measurements indicate a mixed water column. South of Heron Island, the current speeds approached, but did not always exceed, the required speed as determined by the Simpson-Hunter parameter. Here, the development of stratification can be matched to the periods of lower current. However, there are periods where the water column appears well-mixed and thus the stratification does not last.

\section{CONCLUSIONS}

The temperature and current profiling data taken by two moorings in the vicinity of Heron Island verify that during certain conditions, there is sufficient turbulent kinetic energy to create complete vertical mixing of the water column, thereby destroying stratification. Further, the mixing conditions can be correlated to tidal currents. The mooring data were used to empirically determine a Simpson-Hunter value of 2.2 as an index for tidal front formation, and the subsequent analysis assumes that this value is appropriate throughout the coverage area. The Simpson-Hunter parameter, coupled with the bathymetry data, provides an approximate threshold for surface current speed above which tidal mixing should occur. Surface current speed measured by the twin HF radar systems can then be compared to the threshold values determined throughout the radar coverage area. Regions in which the surface currents exceed the threshold value are determined as well-mixed. Regions in which the surface currents do not exceed the threshold value are assumed to have some degree of stratification. The extent of mixing or stratification can then be used as an indication for bleaching. This paper shows that HF Ocean Radar surface current data can be used to indicate conditions under which vertical mixing due to turbulent kinetic energy will occur, distributing surface heat and thus mitigating potential coral bleaching.

\section{ACKNOWLEDGMENTS}

Funding for this work is provided through NOAA Australian Research Council Linkage Grant LP0562157 led by Ove Hoegh-Guldberg, University of Queensland. Infrastructure was provided through the Integrated Marine Observing System (www.imos.org.au) Great Barrier Reef Ocean Observing System Node, funded by the Queensland State Government and the Australian Government's National Collaborative Research Infrastructure Strategy and the Super Science Initiative. The manuscript contents are solely the opinions of the authors and do not constitute a statement of policy, decision, or position on behalf of NOAA or the U. S. Government. The authors also thank Daniel Atwater for his valuable suggestions.

\section{REFERENCES}

[1] Berkelmans, R., Time-integrated thermal bleaching thresholds of reefs and their variations on the Great Barrier Reef, Mar. Ecol. Prog. Ser., 229, 73$82,2002$.

[2] Skirving W.J., M.L. Heron and S.F. Heron, The hydrodynamics of a bleaching event: Implications for management and monitoring, pp.145161 in: Phinney, J.T., et al. (eds) Coral Reefs and Climate Change: Science and Management. AGU Coastal and Estuarine Series, Vol. 61, 350 p. ISBN 0-87590-359-2, 2006.

[3] Skirving, W.J., S.F. Heron, C.R. Steinberg, C. McLean, B.A.A. Parker, C.M. Eakin, M.L. Heron, A.E. Strong, and L.F. Arzayus. 2010. Determining Thermal Capacitance for Protected Area Network Design in Palau. Silver Spring, MD: NOAA Coral Reef Conservation Program. NOAA Technical Memorandum CRCP $12.317 \mathrm{pp}$.

[4] Simpson, J.H. and J.R. Hunter (1974) Fronts in the Irish Sea. Nature, 250, 404-406. 
[5] De Silva Samarasinghe, J.R., Transient salt wedges in a tidal gulf: A criterion for their formation, Estuarine, Coastal and Shelf Science, 28, 129-148, 1989.

[6] Mavor, T.P. and J.J. Bisagni, Seasonal variability of sea-surface temperature fronts on Georges Bank, Deep-Sea Research II 48(2000) 215-243. 